\title{
Uncertainty Quantification of Treatment Regime in Precision Medicine with an Application in Nonparametric Adaptive Design
}

\author{
Dr. Min-ge Xie \\ Rutgers University, USA \\ mxie@stat.rutgers.edu
}

Personalized decision rule in precision medicine is a 'discrete parameter', for which theoretical development of statistical inference is lacking. This talk proposes a new way to quantify the estimation uncertainty in a personalized decision based on confidence distribution (CD). Suppose, in a regression setup, the optimal decision for treatment versus control for an individual $\mathrm{z}$ is determined by a linear decision rule $D=I\left(m_{-} \_(z)>m_{-} 0(z)\right)$, where $m_{-} 1(z)$ and $m_{-} 0(z)$ are the expectations of potential outcomes of treatment and control, respectively. The estimated D has uncertainty. We propose to find a $\mathrm{CD}$ for $\mathrm{v}=$ $\mathrm{m}_{-} 1(\mathrm{z})-\mathrm{m}_{-} 0(\mathrm{z})$ and compute a 'confidence measure' of the decision $\{\mathrm{D}=1\}=\{\mathrm{v}>0\}$. This measure, with value in $[0,1]$, provides a frequency-based assessment about the decision. For example, if the measure for $\{D=1\}$ is $63 \%$, then, out of 100 patients the same as patient $z, 63$ will benefit using treatment and 37 will be better off in control group. This confidence measure is shown to match well with the classical assessments of sensitivity and specificity, but without the need to know the true $\{D=1\}$ or $\{D=0\}$. Utility of the development is demonstrated in an adaptive clinical trial with nonparametric regression models.

Joint work with Yilei Zhan (Rutgers University) and Sijian Wang (Rutgers University) 